\title{
Delimitação de uma área multidisciplinar para análise bibliométrica de produção científica: o caso da Bioprospecção
}

\author{
Delimitation of a multidisciplinary area for \\ bibliometric analysis of scientific production: \\ study case in the area of Bioprospecting
}

Ricardo Arcanjo de LIMA'

Lea Maria Leme Strini VELHO ${ }^{2}$

Leandro Innocentini Lopes de FARIA ${ }^{3}$

\section{RES U MO}

Os indicadores bibliométricos em ciência e tecnologia surgem da necessidade de mensuração da produção das instituições científica, partindo da premissa de que a ciência se encontra, em grande parte, incorporada em sua literatura. Como a ciência torna-se cada vez mais multidisciplinar, uma das principais dificuldades da análise bibliométrica tem sido a construção de indicadores de áreas com essa característica. Isso ocorre porque as bases de dados científicas adotam uma classificação própria de áreas, subáreas e disciplinas científicas e atribuem tais classificações aos periódicos. Este trabalho insere-se nesse contexto, contribuindo especificamente para a delimitação e construção de indicadores de uma área científica de atividade caracteristicamente multidisciplinar, a bioprospecção. Esta área de conhecimento vem se destacando na esfera política e econômica dos últimos anos. Os resultados deste estudo demonstram ainda a importância dessa área no meio acadêmico, ao comprovar o crescimento da área pelo número de artigos publicados, de disciplinas envolvidas, dos principais periódicos e das instituições de pesquisa que se destacam na produção dos conhecimentos multidisciplinares da bioprospecção.

Palavras-chave: bibliometria; bioprospecção; indicadores científicos.

\section{A B S T R A C T}

The bibliometric indicators in science and technology were born from the need for evaluating the research productivity of the scientific institutions, departing from the premise that knowledge produced in any scientific area is, to a large extent, incorporated in its literature. However, science is becoming increasingly multidisciplinary, and one of the main difficulties in performing the bibliometric analysis has been to construct indicators that would match up with the characteristics of a given multidisciplinary area. This occurs because the scientific databases adopt a classification of knowledge that is specific to each of the scientific areas, sub-areas and

\footnotetext{
1 Bacharel em Biblioteconomia e Ciência da Informação pela Universidade Federal de São Carlos (UFSCar), e Mestrando em Política Científica e Tecnológica pela Universidade Estadual de Campinas (UNICAMP). Correspondência para/Correspondence to: R.A. LIMA. E-mail: <ricardo_bci@yahoo.com.br>.

2 Doutora em Política Científica e Tecnológica pela University of Sussex (1985) e pos-doutorado pelo Ohio State University (1990). Atualmente é professora titular da Universidade Estadual de Campinas (UNICAMP). E-mail: <velho@ige.unicamp.br>.

${ }^{3}$ Doutor em Sciences de L'information Et Communication pela Universite d'Aix-Marseille III (Droit, Econ. et Sciences). Atualmente é professor adjunto da Universidade Federal de São Carlos (UFSCar).E-mail: <leandro@nit.ufscar.br>.

Recebido em 4/1 1/2006 e aceito para publicação em 21/5/2007.
}

TransInformação, Campinas, 19(2):153-168, maio/ago., 2007 
disciplines, attributing as well the same classification to the scientific periodicals. This study, developed within such context, contributes specifically to the delimitation and construction of indicators for an area of multidisciplinary scientific activity, the bioprospecting. In the last years this area of knowledge has burgeoned in the economical and political spheres. This study demonstrates that bioprospecting has been also important in the academic milieu. Herein, the findings indicate a markedly growing interest on the subject, made evident by the number of published articles, academic disciplines, main periodicals and research institutions that distinguished themselves in the knowledge production of such multidisciplinary area.

Keywords: bibliometry; bioprospecting; scientific indicators.

\section{NTRODUÇÃ O}

Uma das normas fundamentais da ciência é que o pesquisador tem que divulgar seus resultados de pesquisa (Merton, 1979). Como conseqüência, a ciência se encontra, em grande parte, incorporada na sua literatura, entendida esta em sua concepção mais ampla. Este reconhecimento de que a atividade científica pode ser recuperada e estudada a partir da sua literatura, ou seja, do seu sistema de comunicação formal, contribuiu, por um lado, para o avanço da história e da sociologia da ciência e, por outro, forneceu a base teórica para a construção de indicadores bibliométricos de produção científica ${ }^{4}$.

Desde meados dos anos 60, quando foram concebidas as premissas conceituais da bibliometria e desenhadas suas principais técnicas e fontes de informação ${ }^{5}$, ela tem sido usada para os mais variados objetivos. Estes últimos incluem: traçar as tendências de crescimento de áreas de conhecimento; detectar o surgimento de novas disciplinas científicas; estimar a contribuição de países e instituições para a produção científica mundial; identificar os principais periódicos de uma área; além de avaliar o desempenho científico de países, instituições e grupos de pesquisa (Spinak, 1998). Para a consecução de cada objetivo desejado, faz-se necessária a construção de indicadores bibliométricos específicos que sejam capazes de refletir, de maneira reconhecidamente válida, a relação entre o conceito em análise e a medida empregada (Nederhof, 1988).

Tais indicadores podem ser, em linhas gerais, classificados como indicadores de atividade e indicadores de ligação. Os primeiros são criados a partir da contagem de publicações e visam à elaboração de listas de freqüência de produção ou ranking de grupos de pesquisa, instituições, empresas e países (Faria, 2001). Este tipo de indicador permite identificar os autores mais produtivos de uma determinada área, analisar sua evolução no decorrer dos anos e mapear quais são os temas de pesquisa prioritários de cada país, dentre outras possibilidades.

Já os indicadores de ligação "têm sido aplicados para elaboração de mapas descritivos do conhecimento e de redes de relacionamento entre pesquisadores, instituições e países" (Faria 2001). Eles são importantes na compreensão da dinâmica de produção do conhecimento científico, pois identificam e quantificam as relações entre os diversos campos que compõem uma base de dados. Por exemplo, os indicadores de ligação possibilitam detectar parcerias entre autores, instituições e países, assim como revelar ligações entre temas ou áreas de pesquisa. Além disso, prestam-se também a fornecer dados para analisar tanto a relação entre pesquisa básica e aplicada, como a intensidade de cooperação entre universidade e empresa (Van Raan; Leeuven, 2002).

Apesar de atender a tantos objetivos diferentes, os indicadores bibliométricos apresentam uma série de limitações, tanto conceituais como metodológicas. Quanto às primeiras, argumenta-se que os indicadores bibliométricos só contemplam a informação científica registrada na literatura, excluindo parte importante da atividade científica, tal como o conhecimento tácito, transmitido diretamente na formação do pesquisador (Davył; Velho, 2000). Ainda mais significativo é o fato de que tais indicadores assumem que a publicação científica está inexorável e positivamente relacionada com o sistema de recompensa vigente na ciência. Sabese, entretanto, que esse nem sempre é o caso, principalmente em países em desenvolvimento (Cozzens, 1989; Luukkonen, 1997).

\footnotetext{
${ }^{4}$ A racionalidade dos indicadores se expressa na seguinte premissa: "o fato de a ciência ser quase sempre publicada de uma forma ou de outra permite usar o número e a qualidade destas publicações como indicador". (Morita-Lou, 1985)

5 Para uma reconstituição histórica do surgimento e evolução da bibliometria, ver Velho (1994).
} 
Com relação às limitações metodológicas, há inúmeras críticas à fonte dos dados usados na construção dos indicadores, ou seja, às bases de dados internacionais. Alega-se que essas bases representam essencialmente a ciência do primeiro mundo, publicada em idioma inglês e em periódicos de alta reputação (Sayão, 1996). Além do viés anglófono - e da representação dominante das publicações norte-americanas no caso específico do Science Citation Index - a própria estrutura dessas bases é alvo de críticas, já que elas foram construídas, não para avaliação e medição da produção científica, mas para facilitar os processos de identificação e recuperação de publicações (Adam, 2002).

Apesar das críticas a esta bibliometria centrada em bases internacionais, ela tem sido amplamente usada, pois, além da facilidade de acesso, possibilita a análise de um grande volume de dados quantitativos. Isso confere um grau de confiabilidade e replicabilidade à informação, que não se obtém com o uso de instrumentos ad hoc de coleta de dados.

importante, quando se usam as ferramentas bibliométricas e as bases de dados internacionais, é reconhecer os limites das mesmas para os objetivos propostos. Esse reconhecimento implica fazer os ajustes metodológicos necessários, tornando-os explícitos e levando-os em consideração no momento da análise dos resultados, de modo a evitar conclusões "muito fortes", sem sustentação empírica adequada.

Uma das principais dificuldades da análise bibliométrica é como recuperar, a partir do universo da base de dados, as informações relevantes para o objeto de estudo que se pretende. Isso ocorre porque as bases de dados internacionais adotam uma classificação própria de áreas, sub-áreas e disciplinas científicas, atribuindo essas classificações aos periódicos de acordo com critérios que têm sido bastante contestados (Glanzel; Schubert, 2003). Conseqüentemente, a análise bibliométrica, mesmo em áreas tradicionais do conhecimento, encontra dificuldades para delimitar o campo a ser estudado (Lewinson, 1999). O problema ganha muito maior magnitude quando se trata de áreas multidisciplinares. Dado que poucas áreas interdisciplinares têm seus próprios periódicos, e dada a tendência dos autores de publicarem em periódicos representativos de suas especialidades (Glanzel; Schubert, 2003), a delimitação das publicações de áreas de pesquisa multidisciplinares tem sido considerada um dos grandes desafios metodológicos da bibliometria (Zipp, 1999).

Este artigo busca dar uma contribuição metodológica a exatamente essa questão, ou seja, como delimitar dentro da base de publicações do Science Citation Index uma área multidisciplinar; neste caso, a área de bioprospecção. Delimitar, dentro de uma base de publicações, uma área multidisciplinar, para análise bibliométrica, significa definir os limites de tal área a partir da identificação dos artigos científicos que sejam reconhecíveis, pelos praticantes desta área, como contendo resultados de pesquisas que são de seu interesse direto (Rogers; Anderson, 1993). Mais especificamente para o nosso caso, delimitar a área de bioprospecção no Science Citation Index foi um exercício de definir os critérios pelos quais alguns artigos dentro da área disciplinar de Química de Produtos Naturais, por exemplo, deveriam ser incluídos na área multidisciplinar de bioprospecção e outros, não. De modo mais geral, delimitar significa, então, identificar e recuperar de uma base apenas os artigos que sejam pertinentes a certa área de pesquisa.

Para tanto, apresenta-se, em primeiro lugar, uma definição desta área, com uma descrição sucinta das atividades que ela contempla, assim como as razões pelas quais ela tem-se tornado uma área importante de pesquisa e, portanto, de publicações. Em seguida, faz-se um relato da metodologia construída para a coleta dos dados, contendo uma exposição detalhada dos passos seguidos no estudo, com o objetivo de servir de base a outros trabalhos bibliométricos que possam ser empreendidos em áreas multidisciplinares. Por fim, apresenta-se a análise propriamente dita da produção científica na área de bioprospecção, a partir de dados extraídos do Science Citation Index no período de 1986-2006. Esta análise compreende a identificação das áreas tradicionais de conhecimento que mais contribuem para os estudos de bioprospecção, assim como dos periódicos científicos que mais publicam artigos nessa área. Da mesma forma, destacam-se os países que mais contribuem para a produção científica em bioprospecção. Segue-se, então, uma análise das instituições latino-americanas em geral, e brasileiras, em particular, que se destacam como produtoras de conhecimento em bioprospecção.

Dado que este estudo se baseou exclusivamente em dados quantitativos, nem sempre foi possível encontrar explicações para os resultados obtidos. 
Algumas explicações alternativas, no entanto, serão discutidas sempre que houver informações adicionais que assim o permitam.

\section{CARACTERIZAÇÃO DA ÁREA DE BIOPROSPECÇ ÃO}

O aproveitamento de recursos da biodiversidade para fins econômicos aumentou muito nos últimos anos. Um bom exemplo é o mercado da estatina ${ }^{6}$, que superou os 15 bilhões de dólares no ano de 2004 (Viegas; Bolzoni; Barreiro, 2006). Valores como o apresentado, conseqüentemente, têm atraído a atenção tanto dos países biodiversos, como dos detentores de recursos técnico-científicos, o que, aliado à crescente consciência dessa valoração dos recursos, tem motivado o estabelecimento de regras para a sua exploração. Assim, surgiu em âmbito planetário uma nova atividade econômica, focada na busca de oportunidades de aproveitamento econômico dos recursos naturais biológicos, chamada bioprospecção (Artuso, 2002).

Sendo assim, a bioprospecção pode ser definida como atividade exploratória que visa a identificar componentes do patrimônio genético e informação sobre conhecimento tradicional, com potencial para o uso comercial (Brasil, 2001). As modernas formas de bioprospecção envolvem a aplicação de avançadas tecnologias no desenvolvimento de novos produtos farmacêuticos, agroquímicos, cosméticos, enzimas industriais e outros, oriundos da biodiversidade (Artuso, 2002).

Antes da assinatura da $\mathrm{CDB}^{7}$, os recursos da biodiversidade, em especial os recursos genéticos, eram considerados patrimônios da humanidade, apesar de diversos países, entre eles o Brasil, já adotarem legislação específica sobre o tema.

Qualquer interessado em pesquisar e/ou explorar a biodiversidade poderia fazê-lo sem que fosse necessário ressarcir os países provedores. Essa posição não era aceita por esses países e por grupos ambientalistas, que exigiam alguma forma de compensação ou de divisão dos benefícios obtidos pelas empresas com a comercialização de produtos desenvolvidos a partir dos recursos genéticos "pirateados". (Pavarini, 2000).

Nesse antigo modelo de bioprospecção, a biodiversidade era coletada sem fiscalização, por pesquisadores/técnicos.. Muitas vezes eram extraídas quantidades enormes de material biológico, prejudicando com isso o meio ambiente; como exemplo, o caso da quase extinção da população adulta da planta maytenus buchanni, usada pelo Instituto Nacional do Câncer dos Estados Unidos como fonte do composto anticancerígeno maytansine. Foram coletados 27.215 quilos de maytenus buchanni, para serem usados em testes no programa de desenvolvimento de novas drogas (Reid et al., 1993 apud Pavarini, 2000). Em muitos casos como esse não eram fornecidos retornos das pesquisas ao país de origem da matéria prima. $\bigcirc$ modelo se revelou muito prejudicial ao meio ambiente e aos países detentores de biodiversidade.

A CDB veio regulamentar em escala mundial essas atividades. Um dos objetivos da convenção é garantir a repartição justa e eqüitativa dos benefícios derivados da utilização dos recursos genéticos. A reivindicação dos países biodiversos foi decisiva na aprovação deste objetivo.

Segundo Azevedo (2005), os países biodiversos julgaram injusta a situação na qual, ao mesmo tempo em que se permitia o livre acesso aos recursos genéticos, os produtos obtidos a partir desses recursos poderiam tornar-se objeto de apropriação por empresas sediadas, na maioria dos casos, em países desenvolvidos. A inclusão desse objetivo na CDB permitiu que fosse reconhecida a soberania dos países sobre a sua própria biodiversidade, não sendo mais esta considerada patrimônio da humanidade, e passando esta biodiversidade a ser regulada por legislação nacional.

Como se pode observar, a exploração da biodiversidade é uma atividade complexa e tende a envolver diversos atores como governos, pesquisadores, comunidades indígenas e tradicionais, empresas de diversos setores, universidades, institutos de pesquisa, entre outros.

\footnotetext{
- Substância derivada de fungos, usada no tratamento de diversas doenças, tais como angina, Alzheimer, osteoporose, câncer, doenças do coração e esclerose múltipla...

7 A Convenção sobre Diversidade Biológica (CDB) ocorreu em julho de 1992, sendo o primeiro encontro mundial sobre a conservação e utilização sustentável da biodiversidade, contando com 175 países signatários.
} 
Essa multiplicidade também ocorre com as diversas áreas de conhecimento que a temática da bioprospecção abrange. Para Trigueiro (2006), no âmbito da pesquisa tipicamente científica e tecnológica, bioprospecção trata-se, por exemplo, das atividades de classificação das espécies, da identificação de suas características morfológicas e fisiológicas de seus componentes genéticos e de suas propriedades ativas, das relações estabelecidas entre eventuais descobertas e conhecimentos antigos e assim por diante. Devido a essa abordagem, pesquisas na área de bioprospecção podem envolver competências de diversas áreas do conhecimento, tais como agronomia, biologia, microbiologia, química, informática, ciências sociais, engenharia genética, direito, farmácia, entre outras.

Esse caráter multidisciplinar da bioprospecção torna complexa a realização de estudos como o presente, que buscam mapear a produção científica na área.

Muitas vezes, essa questão se agrava quando os próprios pesquisadores da área não identificam os resultados de suas atividades como frutos de bioprospecção, tornando muito mais difícil a recuperação dessas pesquisas em bases de dados especializadas. Assad e Sampaio (2005) realizaram buscas na plataforma Lattes do $\mathrm{CNPq}^{8}$ de pesquisadores brasileiros, usando a palavra-chave, bioprospecção. No momento da pesquisa, a plataforma Lattes contava com o total de 390.699 pesquisadores cadastrados. Entretanto, os autores recuperaram apenas 233 nomes, ou seja, apenas $0,06 \%$ do total de pesquisadores; isto nos leva a crer que muitos pesquisadores ainda não identificam suas atividades também como bioprospecção. O problema existe, devido ao reconhecimento relativamente recente desta área multicisciplinar, razão pela qual o uso do termo bioprospecção ainda não está consolidado nas diversas áreas de conhecimento envolvidas. Em tais áreas, esta atividade, recobre outras, que já tinham nomes específicos: um pesquisador da área de química, por exemplo, tradicionalmente usaria o termo "drug discovery", e não "bioprospecção", para definir a sub-área em que ocorreu sua atividade. Identificar outros termos relacionados à bioprospecção para então fazer-se o mapeamento desta área multidisciplinar, foi uma das tarefas mais árduas na realização deste estudo.

\section{PROCEDIMENTOS METODOLÓGICOS}

\section{Base de Dados}

Para a realização do estudo foram coletados dados do Science Citation Index (SCI) via Portal Periódico da Capes?.

O Science Citation Index (SCI) é considerado uma das bases científicas multidisciplinares mais abrangentes e com rigorosos critérios de qualidade adotados pelo Thomson Scientific Information ${ }^{10}$, empresa responsável por sua criação, manutenção e alimentação.

\section{Formulação da Estratégia de Busca}

A formulação de uma estratégia para a recuperação de publicações sobre um tema científico, deve ser realizada criteriosamente para não comprometer a análise da produção científica. Idealmente, para que da análise resulte um panorama fiel sobre o tema, a expressão de busca deve proporcionar a recuperação de todas as publicações relevantes sobre o tema, presentes na base de dados utilizada, e, ao mesmo tempo, evitar a recuperação de publicações não-relevantes. No entanto, tal situação dificilmente é alcançada, principalmente no caso de temas multidisciplinares, que recebem contribuição de diversas áreas do conhecimento.

Como explicado acima, o tema de pesquisa, bioprospecção, é multidisciplinar, recebendo contribuições das áreas de biologia, botânica, zoologia, química, agronomia e várias ciências sociais. Trata-se, também, de um tema relativamente novo, com vocabulário próprio ainda em consolidação, o que leva a uma baixa recuperação de publicações nas buscas que utilizam palavras-chave.

\footnotetext{
8 A Plataforma Lattes é um conjunto de sistemas de informações, bases de dados e portais Web voltados para a gestão de Ciência e Tecnologia (C\&T). Foi concebida para integrar os sistemas de informações das agências federais, racionalizando o processo de gestão de C\&T.

9 <http://www.periodicos.capes.gov.br>.

10 Antigo Institute for Scientific Informatio (ISI).
} 
A solução encontrada para a recuperação de um conjunto significativo e coerente de publicações que permitisse a análise da produção científica da área de bioprospecção foi a elaboração de uma expressão de busca complexa, composta por grande conjunto de palavras-chave testadas e selecionadas. Este processo de definição da expressão de busca para a delimitação do tema bioprospecção na base de dados Science Citation Index é uma das contribuições deste artigo e pode ser extrapolado para outros temas e bases de dados.

O procedimento adotado foi o seguinte: 0 primeiro passo foi a definição do que realmente recuperar, como identificar os artigos ligados à bioprospecção. Assim, apesar de bioprospecção ser definida comumente como atividade de prospecção da biodiversidade com fim comercial, consideramos bioprospecção toda e qualquer atividade de prospecção biológica, ou seja, usamos na formulação da estratégia de recuperação dos dados, verbos de atividade prospectiva associados a elementos biológicos e da biodiversidade, que podem ser observados detalhadamente nos Quadros 1 e 2.

A dispersão de trabalhos de bioprospecção em diversas áreas do conhecimento era outra questão a

Quadro 1. Estratégias de recuperação no SCl - 1945-2006.

\begin{tabular}{lc}
\hline \multicolumn{1}{c}{ Expressão } & Resultado em registros \\
\hline $\mathbf{1 1 T S}=$ ((prospect* or extract* and (phytochemical or medicinal plant* or phytoterapic* or & 100.000 \\
natural products or organic synthesis) & 23.330 \\
$\mathrm{TS}=$ (natural products or botanical products or medicinal plants or herbs or herbal medicines & \\
or phytotheraphy or phytomedicines or phytomedicinals or phytochemical substances or & \\
phytopharmaceutic products or aromatic oils or essential oils or fatty oils or vegetable oils & \\
or bioactive constituents or bioactive substances or plant pharmacology) & 24.048 \\
$\mathrm{TS}=$ (natural products or plant extracts or botanical products or medicinal plants or herbs or \\
herbal medicines or phytotheraphy or phytomedicines or phytomedicinals or phytochemical \\
substances or phytochemistry substances or phytopharmaceutic products or aromatic oils or \\
essential oils or fatty oils or vegetable oils or bioactive constituents or bioactive substances \\
or plant pharmacology)
\end{tabular}

Quadro 2. Estratégias de recuperação no SCl - 1945-2006.

\begin{tabular}{|c|c|}
\hline Expressão & Resultado em registros \\
\hline $\begin{array}{l}\text { TS }=(=(((\text { ethnobotan* or ethnopharm* or bioprospect* or indi* knowledge or traditional } \\
\text { knowledge) or (prospection or prospect or extract and (plant-derived drugs or bio* or } \\
\text { microorganism* or microbi*or molecular-genetic* or genetics-plant or molecular biology or } \\
\left.\left.\left.\left.\text { plant* or animal* }{ }^{*}\right)\right)\right)\right)\end{array}$ & 32.875 \\
\hline $\begin{array}{l}\text { TS }=(((((\text { ethnobotan* or ethnopharm* or bioprospect* or indi* knowledge or traditional } \\
\text { knowledge or biodiversity prospecting) or (prospection or prospect or extract and (plant-derived } \\
\text { drugs or bio* or microorganism* or microbi*or molecular-genetic* or genetics-plant or } \\
\left.\left.\left.\left.\text { molecular biology or plant* or animal* })^{*}\right)\right)\right)\right)\end{array}$ & 32.890 \\
\hline $\begin{array}{l}\mathrm{TS}=(((((\text { ethnobotan* or ethnopharm* or bioprospect* or indi* knowledge or traditional } \\
\text { knowledge or biodiversity prospecting) or (prospection or prospect or extract and (plant-derived } \\
\text { drugs or microorganism* or microbi* or molecular-genetic* or genetics-plant or molecular } \\
\left.\left.\left.\left.\left.\text { biology or plant }{ }^{*}\right)\right)\right)\right)\right)\end{array}$ & 18.863 \\
\hline
\end{tabular}

11 Topic Search, tópico de pesquisa/assunto (títulos, resumos, palavras-chave).

TransInformação, Campinas, 19(2):153-168, maio/ago., 2007 
ser respondida, assim como recuperar os registros de artigos sobre bioprospecção presentes nas mais diversas áreas cobertas pelo $\mathrm{SCl}$.

Inicialmente procuramos construir as estratégias na modalidade de busca do SCl - General Search, usando palavras-chave da literatura disponível e presente na Plataforma-Lattes de pesquisadores que realizam algum tipo de atividade em bioprospecção.

Baseados nesses dados, formulamos as primeiras expressões de recuperação dos dados, conforme o Quadro 1.

Essas expressões de busca tinham apenas um caráter exploratório, ou seja, servir de subsídio para a formulação de expressões mais refinadas e avaliar como a área está organizada na base de dados. Na etapa seguinte, inserimos palavras-chave ligadas a outros contextos da bioprospecção como, conhecimento tradicional, genética e biologia molecular.

Essas expressões ainda não eram suficientemente precisas, além de não recuperar documentos indexados a partir de outros contextos, mas também serviram como subsídio ao refinamento das palavras usadas nas futuras expressões. Nessa fase, ficou evidente a tensão entre tratar de incluir toda a área e a necessidade de delimitar sub-áreas para não "poluir" a informação.

Portanto, os registros recuperados a partir das expressões do Quadro 2 sofreram tratamento bibliomé- trico, onde foi possível fazer uma classificação das principais palavras ligadas à bioprospecção e, a partir daí, trabalhar com expressões mais focadas. Assim, começamos a trabalhar com as palavras expostas no Quadro 3.

Com a expressão de busca (Quadro 3) aplicada apenas ao título, foram encontrados 17.271 artigos. A relevância dos artigos nos pareceu satisfatória, mas possivelmente muitos artigos relevantes ficaram de fora. A mesma expressão aplicada ao registro completo resultou em mais de 100.000 registros.

A partir da experiência com as expressões anteriores, verificamos que o melhor caminho seria a combinação de diferentes expressões. Para isso, fizemos uso da modalidade de busca avançada do $\mathrm{SCl}$, o Advanced Search. Executamos diversas expressões de busca no $\mathrm{SCl}$ e as combinamos com outras expressões, recuperando assim 25.258 registros, sendo que destes 20.439 eram artigos.

Assim, tendo como base para a recuperação de artigos a definição de bioprospecção como toda atividade de prospecção biológica, assumimos que as expressões combinadas, presentes no Quadro 4, são suficientemente precisas para a recuperação de artigos ligados à bioprospecção, necessários para a execução deste estudo.

Uma vez definida a estratégia, foi possível realizar a coleta de dados e o tratamento bibliométrico para a construção dos indicadores.

Quadro 3. Palavras Refinadas.

\begin{tabular}{ll}
\hline Verbos & $\begin{array}{l}\text { Palavras Refinadas } \\
\text { sensing, research*, prospect* }\end{array}$ \\
Foco & $\begin{array}{l}\text { marine, flora, plants, fauna, micro-organisms, animals, microbial, bacteria, virus, } \\
\text { herbal, fungal, soil, fungus, insects, fungi, species, mammalian, bioresources, } \\
\text { natural product, genetic, genomic, biochemical, medicinal, pharmaceutical }\end{array}$ \\
Ethnobotany, ethnopharmacology, indigenous knowledge OR traditional knowledge, \\
Ethnomedicinal, Bioprospecting, biodiversity prospection, Biodiscovery
\end{tabular}


Quadro 4. Buscas combinadas (período - 1986-2006).

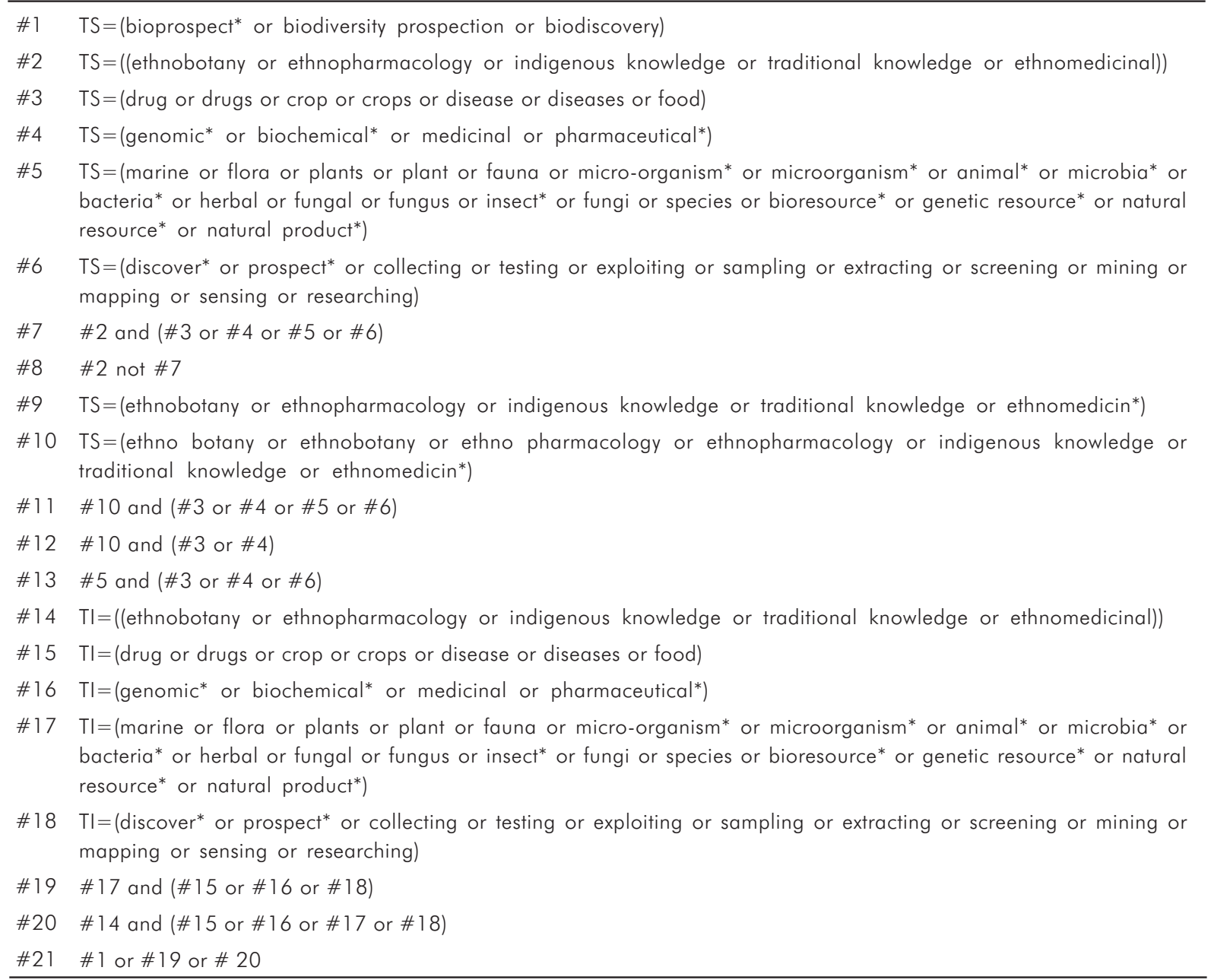

Para o tratamento bibliométrico foi utilizado o software VantagePoint, que nos possibilitou trabalhar automaticamente com cada um dos campos da base, listando e agrupando as informações contidas nos registros. $\bigcirc$ VantagePoint foi utilizado por sua capacidade de tratar grandes volumes de dados.

\section{RESULTADOS}

A partir dos dados coletados no $\mathrm{SCl}$, foram produzidos indicadores bibliométricos que nos vão ajudar a compreender a atividade científica na área de bioprospecção. A Figura 1 apresenta o número de publicações indexadas anualmente na área de bioprospecção desde 1986.

gráfico nos mostra que, com algumas variações, tem havido um crescimento constante do número de publicações na área de bioprospecção desde o ano de 1986. Uma das causas prováveis desse crescimento parece ser o papel de destaque que as atividades em bioprospecção vêm ganhando em escala global nos últimos anos, principalmente como resultado da implementação da CDB, conforme mencionado acima. Para implementar as atividades previstas na CDB houve, em vários países, um fluxo maior de investimentos em programas de pesquisa relacionados com a prospecção biológica. Nos Estados Unidos, por exemplo, os Institutos Nacionais de Saúde (NIH), em cooperação com a National Science Foundation e a Agência Americana de Cooperação para o Desenvolvimento (USAID), criaram, em junho de 1992, o International Cooperative Biodiversity Groups (ICBG), consórcios que cobrem áreas relacionadas à conservação da biodiversidade, coleta de plantas, 
desenvolvimento de drogas e gerenciamento e disseminação de informação. (Sant'ana, 2002). Este programa, ainda em vigência, já realizou cinco chamadas públicas e seleção de projetos. Como os projetos foram iniciados em 1994, e a publicação de resultados na forma de artigos leva algum tempo, é possível que a taxa de crescimento de publicações em bioprospecção reflita, pelo menos em parte, as atividades de pesquisa realizadas no âmbito deste programa. É importante apontar ainda que, de 1994 até agora, portanto, durante 12 anos, foram alocados cerca de 6 milhões de dólares americanos por ano para esse programa, levando ao total de aproximadamente 72 milhões de dólares, uma quantia nada trivial para uma área de conhecimento ainda em formação.

Cabe ressaltar que o aparente declínio do número de artigos apontados em 2006 se deve ao fato da coleta dos dados ter sido feita em maio de 2006, e não a uma queda real no número de artigos indexados.

Figura 2 nos mostra a classificação dos registros indexados feita pelo $\mathrm{SCl}$. Podemos observar que a maioria dos artigos se encontra nas áreas das chamadas ciências biológicas e agrárias, assim como biologia, medicina, farmácia, química e agronomia, com destaque para áreas específicas como a microbiologia, ecologia/meio ambiente, genética/biologia molecular e imunologia.

A predominância dessas áreas já era esperada devido às próprias características da bioprospecção; mas é interessante ressaltar que áreas de pesquisa mais novas, como a genética e a biologia molecular, têm uma participação significativa perante as tradicionais áreas químico-farmacêuticas. Isso, provavelmente evidencia o papel de crescente importância das técnicas biotecnológicas, para o desenvolvimento e geração de produtos originados da biodiversidade. Nota-se, também, que um número considerável de registros está classificado como multidisciplinar, confirmando que a pesquisa em bioprospecção, como se afirmou anteriormente, reúne pesquisadores de diversas áreas de conhecimento.

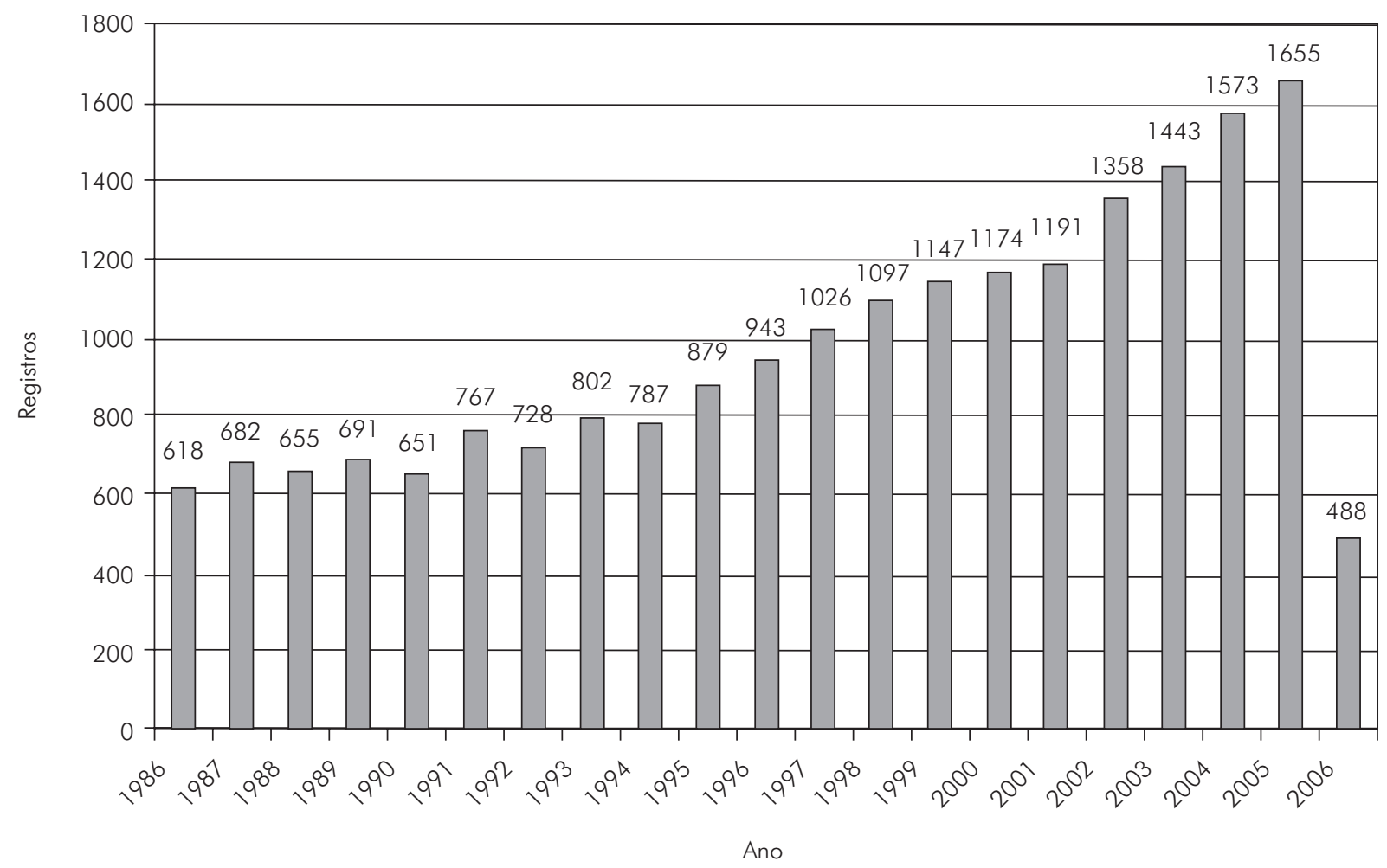

Figura 1. Número de artigos indexados por ano no SCl na área de Bioprospecção no período de 1986-2006. Fonte: SCI/ISI, via Web of Science (2006). 
Observa-se também no Figura 2 a presença de registros nas áreas de ciências dos materiais, física e matemática. Ainda que esse número seja pequeno cerca de 200 artigos em um período de 20 anos - não era esperado que essas áreas contribuíssem para a pesquisa em bioprospecção. A explicação é que essas classificações não são baseadas nos registros individuais e sim no periódico onde o artigo foi publicado, o que pode ter acarretado alguma distorção nos resultados.

Outro resultado digno de nota é a baixa representatividade em número de artigos das áreas de ciências sociais. Sabe-se que as ciências sociais, particularmente a Antropologia, desempenham papel fundamental em estudos que ligam a bioprospecção à atividade humana, tal como o conhecimento tradicional adquirido da biodiversidade por grupos populacionais nativos. Por essa razão, acredita-se que as ciências sociais estejam sub-representadas nos nossos resultados em conseqüência do uso do Science Citation Index como fonte da informação. Isto porque essa base de dados indexa apenas periódicos das chamadas ciências duras (hard sciences), abrangendo as áreas de Agricultura e Tecnologia dos Alimentos, Astronomia, Ciências do Comportamento, Bioquímica, Biologia, Ciências Médicas, Química, Computação, Eletrônica, Engenharia, Ciências Ambientais, Genética, Geociên-cias, Materiais, Microbiologia, Matemática, Ciências Nucleares, Farmacologia, Física, Estatística, Veterinária, Zoologia e outras áreas afins. (Thomson, c2004). As ciências sociais são cobertas pelo Thomson Scientific no Social Sciences Citation Index, base de dados que é constantemente questionada como fonte de dados para a construção de indicadores. Tal questionamento está relacionado, principalmente, ao fato de que as ciências sociais apresentam um caráter distinto do das chamadas ciências duras, suas temáticas são de maior interesse local, o que leva a um fluxo maior de publicações em revistas nacionais e em outros meios de comunicação (García; Román, 2006)

No Quadro 5 é apresentada a lista dos 20 periódicos que mais publicaram artigos na área de

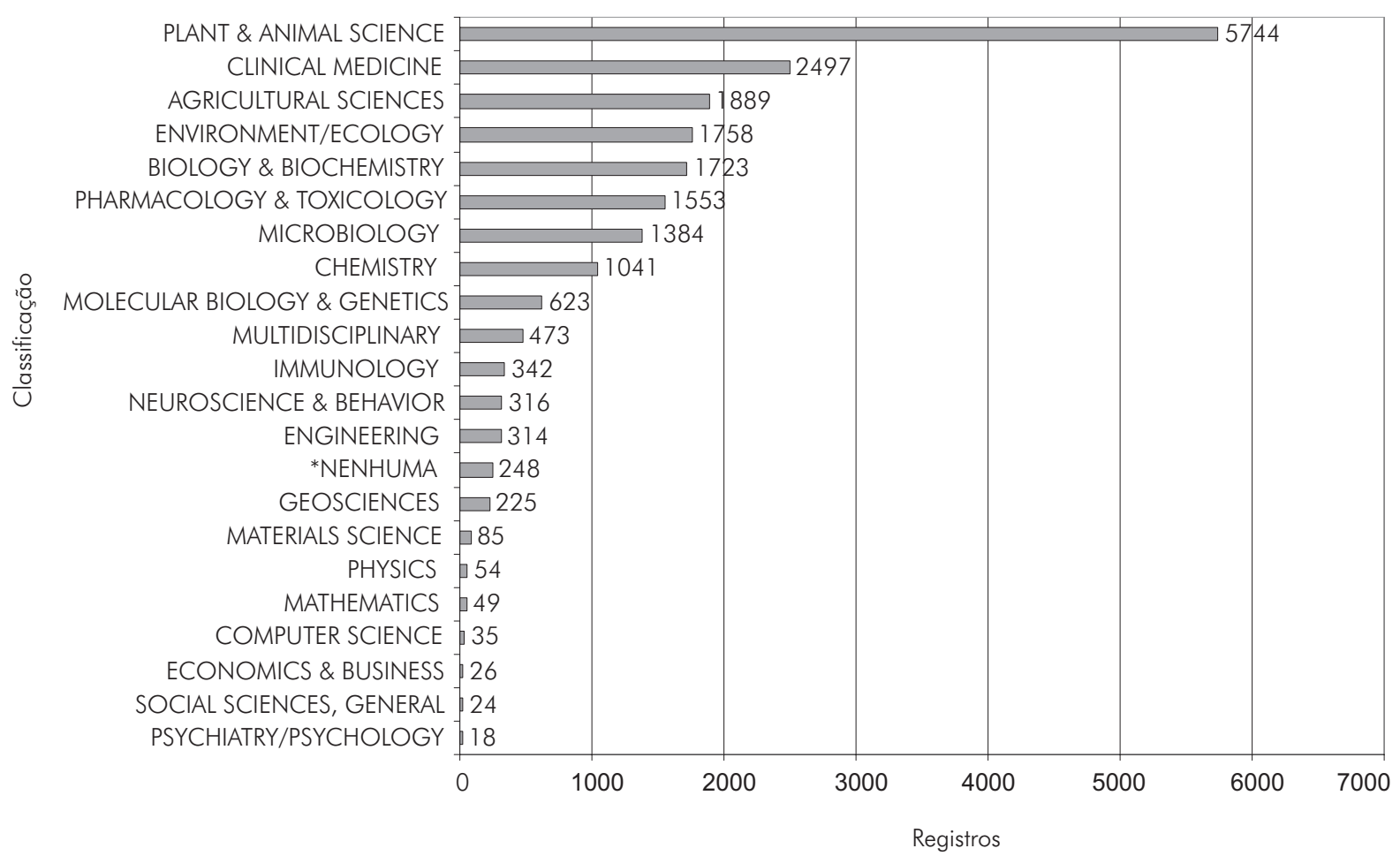

Figura 2. Classificação dos Registros recuperados segundo critérios do SCl no período de 1986-2006.

Fonte: SCI/ISI, via Web of Science (2006) / * Registros que não receberam classificação do SCl.

TransInformação, Campinas, 19(2):153-168, maio/ago., 2007 
bioprospecção. A lista está em conformidade com as classificações apresentadas no Figura 2. Um dado interessante é a liderança do periódico Journal of Ethnopharmacology, que justamente privilegia o exame de informações e conhecimentos ligados ao conhecimento tradicional a respeito de plantas, fungos, animais, microrganismos e minerais, sendo conhecido como um periódico multidisciplinar devotado às "drogas" indígenas.

Ele é seguido por publicações da área de microbiologia, biologia e fitoterápicos, e outras áreas ligadas às ciências biológicas, da vida e afins. É interessante ressaltar a presença de periódicos científicos voltados mais a aspectos práticos e econômicos da biodiversidade como o Economic Botany da Society for Economic Botany e o Journal of Natural Products da American Chemical Society em parceria com a American Society of Pharmacognosy; entretanto, os outros periódicos também abarcam esses aspectos.
A Figura 3 apresenta um ranking das instituições de pesquisa que, no período analisado, mais publicaram artigos na área. Podemos observar uma clara predominância de instituições dos Estados Unidos. Em uma classificação das 23 instituições, 15 delas são dos EUA, com destaque para órgãos governamentais como o USDA ${ }^{12}$ e o USFDA ${ }^{13}$.

Isto indica que provavelmente os EUA consideram a bioprospecção uma atividade estratégica, levando inclusive órgãos governamentais a publicarem na área. Dentre as instituições estadounidenses destacamos a universidade de Cornell e a da Geórgia, ambas participantes de projetos do consórcio ICBG ${ }^{14}$ mencionado acima, o que pode ser uma das razões pelas quais figuram entre as instituições que mais produziram na área.

As outras instituições pertencem à França, Rússia, Brasil, China, Canadá, Bélgica e Espanha. É

Quadro 5. Periódicos que mais publicaram artigo na área de Bioprospecção no período de 1986-2006.

\begin{tabular}{ll}
\hline Registros & \\
\hline 441 & JOURNAL OF ETHNOPHARMACOLOGY \\
147 & JOURNAL OF CLINICAL MICROBIOLOGY \\
135 & APPLIED AND ENVIRONMENTAL MICROBIOLOGY \\
134 & PLANT DISEASE \\
131 & PHYTOTHERAPY RESEARCH \\
117 & PROCEEDINGS OF THE NATIONAL ACADEMY OF SCIENCES OF THE UNITED STATES OF AMERICA \\
99 & PHARMACEUTICAL BIOLOGY \\
91 & JOURNAL OF FOOD PROTECTION \\
88 & JOURNAL OF THE AMERICAN VETERINARY MEDICAL ASSOCIATION \\
88 & PHYTOPATHOLOGY \\
86 & CURRENT SCIENCE \\
83 & ECONOMIC BOTANY \\
80 & THEORETICAL AND APPLIED GENETICS \\
79 & JOURNAL OF ECONOMIC ENTOMOLOGY \\
77 & INTERNATIONAL JOURNAL OF FOOD MICROBIOLOGY \\
76 & CHEMICAL \& PHARMACEUTICAL BULLETIN \\
74 & JOURNAL OF NATURAL PRODUCTS \\
73 & PLANT AND SOIL \\
\hline
\end{tabular}

12 Departamento de Agricultura dos Estados Unidos.

13 Órgão governamental dos Estados Unidos que faz o controle dos (tanto humano como animal) suplementos alimentares, medicamentos (humano e animal), cosméticos, equipamentos médicos, materiais biológicos e produtos derivados do sangue humano.

14 International Cooperative Biodiversity Group. 


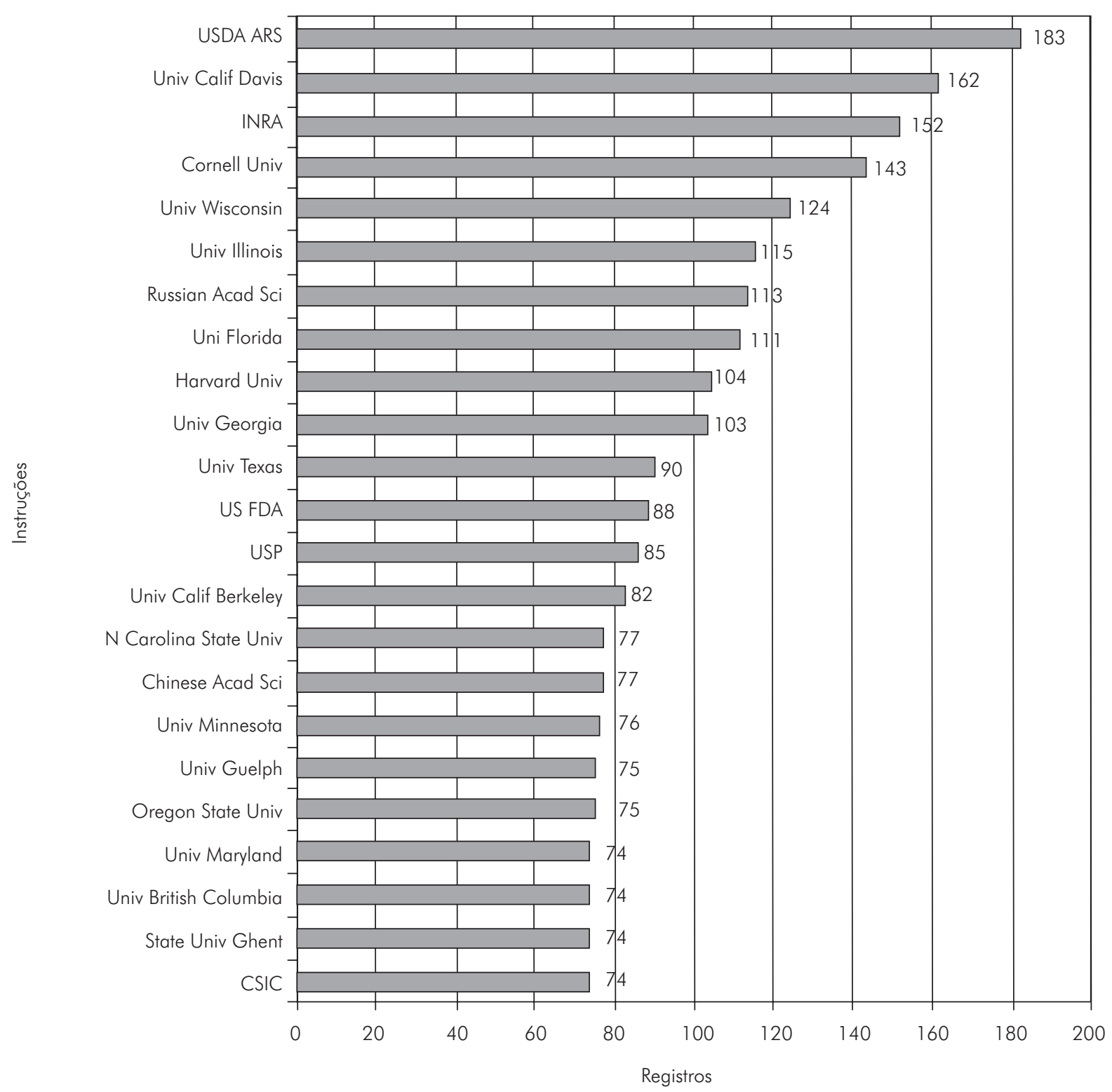

Figura 3. Instituições de Pesquisa que mais publicaram artigos na área de Bioprospecção no período de 1986-2006.

Fonte: SCI/ISI, via Web of Science (2006).

164

interessante notar que entre os países que mais publicam sobre bioprospeção aparecem apenas dois países megadiversos, quais sejam, China, através da Academia Chinesa de Ciências, e Brasil, através da Universidade de São Paulo (USP), que ocupa a $13^{a}$ posição.

A USP é uma das principais instituições de pesquisa envolvidas no Programa Biota da FAPESP, tendo participação em 28 projetos de pesquisa. $\bigcirc$ programa foi instituído em 1999 e tem como objetivo inventariar, caracterizar e definir o potencial econômico da biodiversidade do estado de São Paulo. A participação ativa da universidade em projetos ligados à área de estudo da biodiversidade/bioprospecção explicar a presença da USP entre as instituições que mais publicaram em bioprospecção no período estudado.

A Figura 4 apresenta a participação das instituições latino-americanas no número de publicações. Nele podemos observar uma clara predominância das instituições brasileiras, que respondem por 13 das 20 instituições que mais publicaram no período de 1996-2006, seguidas em volume pela Universidade 
Nacional Autônoma do México (UNAM) e pela Universidade de Buenos Aires (UBA). As demais instituições pertencem a países como o Panamá, Chile, Colômbia e Costa Rica. México, Chile e Costa Rica que estão envolvidos nos projetos ICBG. A Costa Rica, além do ICBG, participa de vários acordos de cooperação internacional através do Instituto Nacional de Biodiversidad - INBIO, centro de referência em gestão e pesquisa da biodiversidade. No caso panamenho podemos destacar a presença do Smithsonian Research
Tropical Institute, centro internacionalmente reconhecido por suas pesquisas ligadas à biodiversidade tropical.

Como as instituições do Brasil se destacam no contexto latino-americano, a Figura 5 faz um recorte no país. Podemos observar que o gráfico enumera as 20 instituições brasileiras que mais publicaram. Observamos que a maioria das instituições está localizada na região Sul/Sudeste e distante das regiões mais biodiversas, como o Centro Oeste/Norte, onde se encontram os ecossistemas do Cerrado, Pantanal e Amazônico.

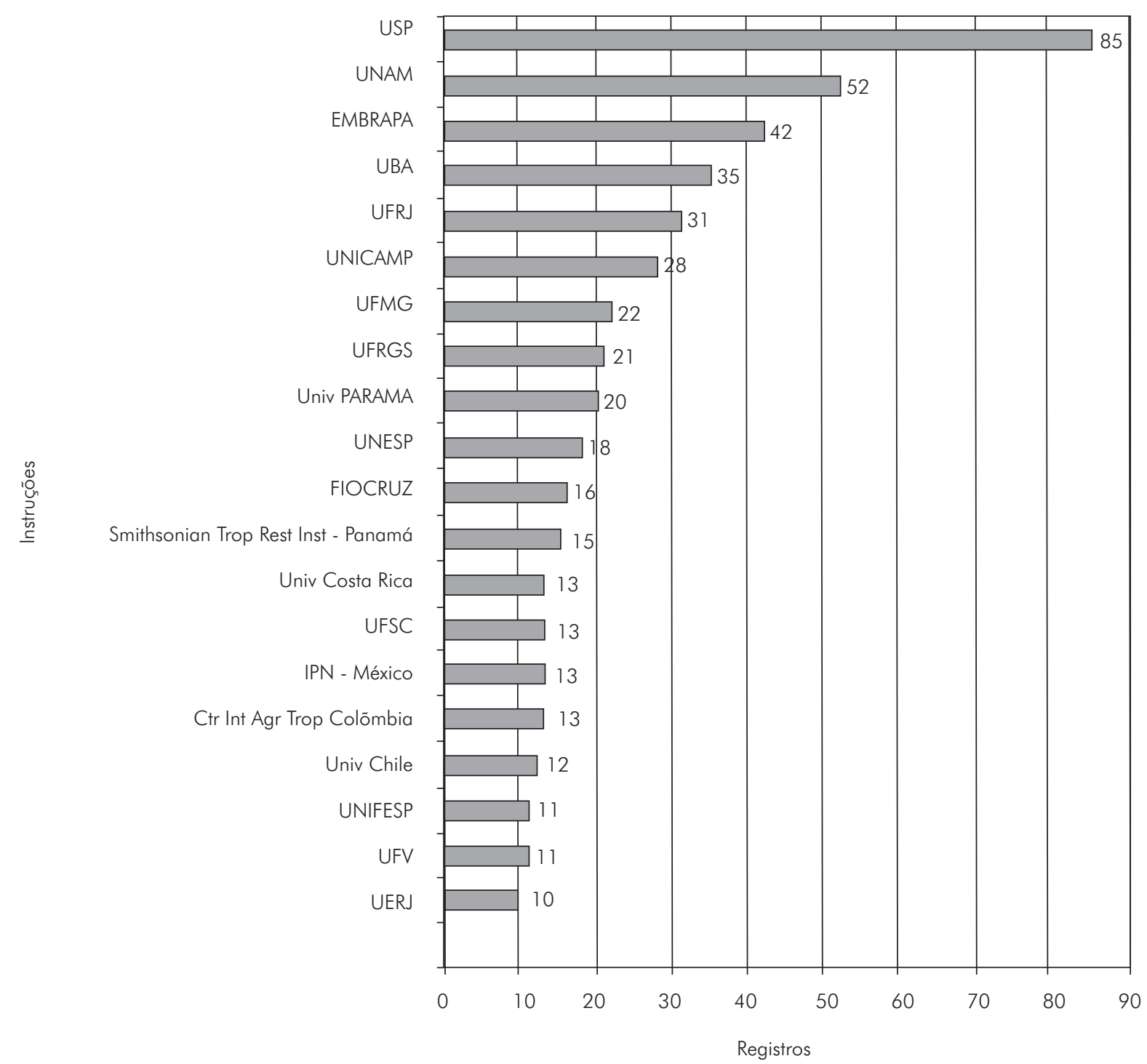

Figura 4. Instituições de Pesquisa Latino-americanas que mais publicaram artigos na área de Bioprospecção no período de 1986-2006.

Fonte: SCI/ISI, via Web of Science (2006). 


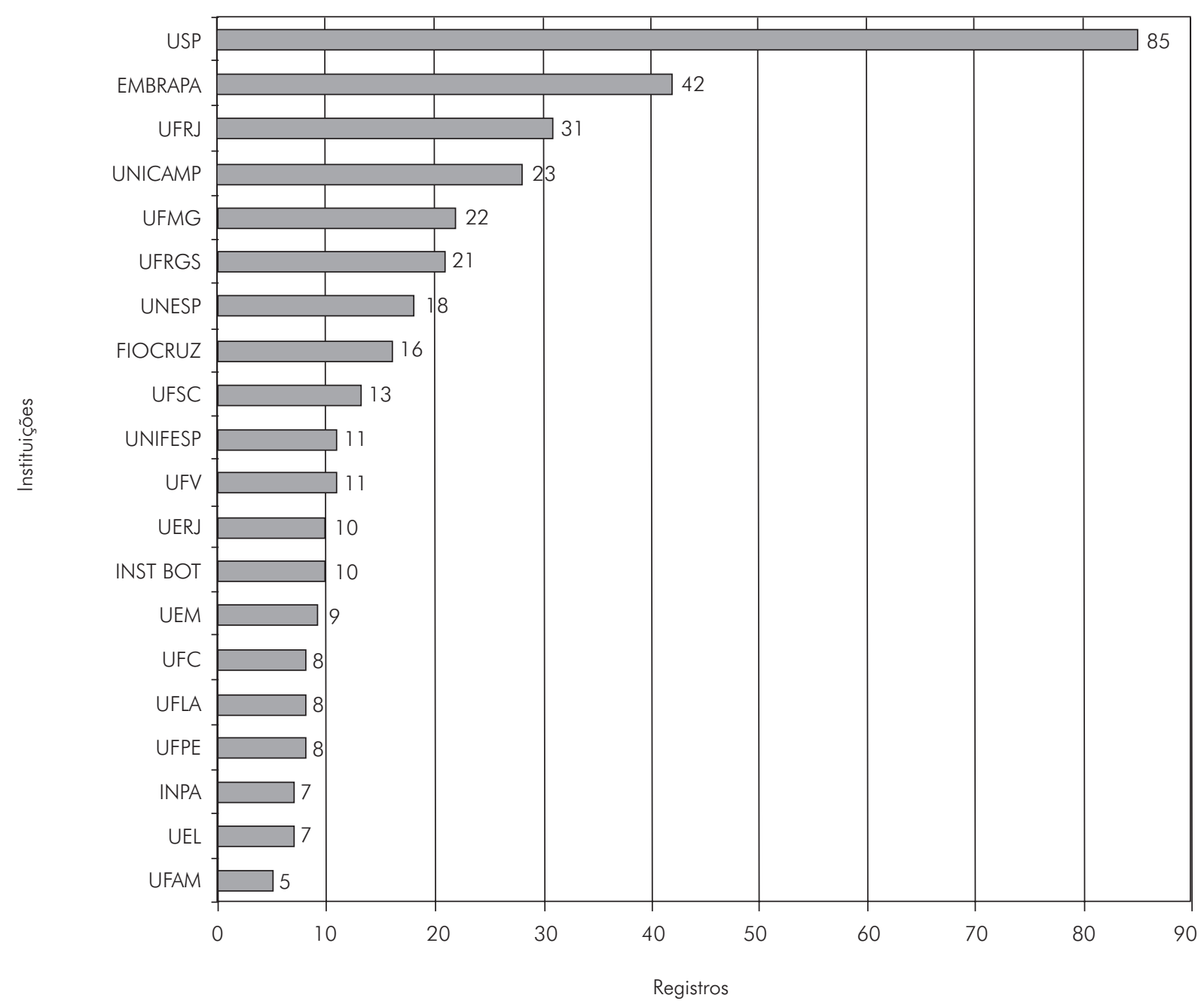

Figura 5. Instituições de Pesquisa Latino-americanas que mais publicaram artigos na área de Bioprospecção no período de 1986-2006.

Fonte: SCI/ISI, via Web of Science (2006).

A pouca participação de instituições das regiões Centro Oeste/Norte, no gráfico apenas representadas pelo Instituto Nacional de Pesquisas da Amazônia - INPA, Universidade Federal do Amazonas - UFAM e pela Empresa Brasileira de Pesquisa Agropecuária - EMBRAPA, que têm centros de pesquisa no cerrado e na Amazônia, é reflexo da alta concentração da pesquisa científica no eixo Sul-Sudeste do país e falta de investimento e desenvolvimento naquelas regiões.

Por outro lado, destaca-se a presença marcante das universidades públicas do estado de São Paulo (USP, UNICAMP e UNESP) que, juntas, respondem por uma considerável fatia da produção científica nacional na área de bioprospecção. Ou seja, dos 370 artigos em bioprospecção produzidos por instituições latinoamericanas nos últimos 20 anos, 131 (cerca de 35\%) se originaram das universidades estaduais de São Paulo.

\section{CONSIDERAÇÕES FINAIS}

A tarefa complexa de delimitar uma área multidisciplinar como a da bioprospecção se mostrou particularmente satisfatória. Através das combinações de expressões já expostas no item 3.2, conseguimos delimitar a área de atividade e assim coletar os dados usados na construção dos indicadores bibliométricos. Desse modo podemos vislumbrar a dinâmica cientíica da área de bioprospecção, área essa que torna-se cada vez mais importante no meio acadêmico. 
número de publicações tem crescido ano a ano em um ritmo veloz, e, apesar da predominância de áreas tradicionalmente vinculadas ao tema, como as áreas químico-farmacêuticas, observamos uma contribuição significativa de áreas como a genética e a biologia molecular. E a tendência é que essas novas áreas continuem crescendo dentro da bioprospecção e passem a contribuir cada vez mais para a pesquisa científica na área, dado o uso cada vez mais intenso das técnicas biotecnológicas.

Os indicadores confirmam a já esperada concentração da produção científica nos chamados países do Norte, em especial nos EUA. Essa concentração reflete a disparidade existente de conhecimento técnico-científico em bioprospecção no "conflito Norte-Sul", ou seja, entre países chamados desenvolvidos e em desenvolvimento.

\section{REFERÊNCIAS}

ADAM, D. Citation analysis: The counting house. Nature, v. 415, feb. 2002.

ARTUSO, A. Bioprospecting, benefit sharing, and biotechnological capacity building. World Development, v.30, n.8, p.1355-1368, 2002.

ASSAD, A.L.D.; SAMPAIO, M.J.A. Acesso à biodiversidade e repartição de benefícios. 2005. Relatório de Pesquisa (DPCT- IG) - Universidade Estadual de Campinas.

AZEVEDO, C.M.A. A regulamentação do acesso aos recursos genéticos e aos conhecimentos tradicionais associados, no Brasil. Biota Neotropica, v.5, n.1, janeiro de 2005. Disponível em: $<$ www. biotaneotropica.org.br/v5n 1/pt/abstract?point-ofview+BN00105012005 >. Acesso em: 28 dez. 2005.

BRASIL. Medida provisória n 2.186-16, de 23 de agosto de 2001. Disponível em: <http://www.mma.gov.br/port/cgen/>. Acesso em: 24 abr. 2006.

COZZENS, S.E. What do citations count? The rhetoric-first model. Scientometrics, v. 15, n.5-6, p.437-447, 1989.

DAVYT, A.; VELHO, L. A avaliação da ciência e a revisão por pares: passado e presente. Como será o futuro? História, Ciências, Saúde-Manguinhos, v.7, n. 1, p.93-116, 2000.

FARIA, L.I.L. Prospecção tecnológica em materiais: aumento da eficiência do tratamento bibliométrico: aplicação na análise de tratamentos de superfície resistentes ao desgaste. 2001. Tese (Doutorado em Ciência e Engenharia dos Materiais) - Programa de Pós-Graduação em Ciência e Engenharia de Materiais, Universidade Federal de São Carlos, São Carlos, 2001.

GLANZEL, W.; SCHUBERT, A. A new classification scheme of science fields designed for scientometric evaluation purposes. Scientometrics, v. 56, n.3, p. 357-367, 2003.

GARCÍA, M.P.G.; ROMÁN, A.R. Impacto de las revistas extranjeras en la geografía española: análisis de citas. Revista Bibliográfica
Essa questão se agrava ainda mais no caso dos países biodiversos que não possuem instrumentos para conhecer e explorar a sua própria biodiversidade. Apesar de constatada a concentração da produção científica em instituições de países do Norte, no recorte feito na América Latina, pode-se observar uma participação expressiva dos países da região, em especial do Brasil.

A bioprospecção é uma área de pesquisa em crescimento e tanto a comunidade científica dos países do Norte como a daqueles do Sul parecem estar interessadas em pesquisar essa temática, o que deve levar cada vez mais à existência de acordos de cooperação e troca de conhecimentos e experiências entre essas comunidades. Analisar as características e a dinâmica dessas parcerias é o foco de outro estudo que estamos empreendendo.

de Geografía y Ciencias Sociales, v. 1 1, n.632, 2006. Disponível em: <http://www.ub.es/geocrit/b3w-632.htm>. Acesso em: 28 nov. 2006.

LEWISON, G. The definition and calibration of biomedical sub-fields. Scientometrics, v.46, n.3, p. 529-537, 1999.

LUUKKONEN, T. Why has latour's theory of citations been ignored by the bibliometric community? Discussion of sociological interpretations of citation analysis. Scientometrics, v.38, p.27-37, 1997.

MERTON, R.K. Os imperativos institucionais da ciência. In: DEUS, J.D. A crítica da ciência. Rio de Janeiro: Zahar, 1979.

MORITA-LOU, H. Science and technology indicators for development. Boulder: Westview Press, 1985.

NEDERHOF, A.J. The validity and reliability of evaluation of scholarly performance. In: VAN RAAN, A.F.J. (Ed.). Handbook of quantitative studies of science and technology. Amsterdam: Elsevier/North-Holland, 1988.

PAVARINI, M.F. Prospecção da diversidade biológica: perspectivas para o caso brasileiro. 2000. Dissertação (Mestrado em Ciências em Planejamento Energético) - Coordenação dos Programas de Pós-graduação de Engenharia, Universidade Federal do Rio de Janeiro, Rio de Janeiro, 2000.

ROGERS, L.A.; ANDERSON, J. A new approach to defining a multidisciplinary field of science: The case of cardiovascular biology. Scientometrics, v.28, n. 1, p.61-67, 1993.

SANT'ANA, P.J.P. É possível a bioprospecção no Brasil. 2002. Tese (Doutorado em Engenharia de Produção) - Coordenação dos Programas de Pós-graduação de Engenharia, Universidade Federal do Rio de Janeiro, Rio de Janeiro, 2002.

SAYÃO, L.F. Bases de dados: a metáfora da memória científica. Ciência da Informação, v. 25, n.3, p.314-318, 1996.

SPINAK, E. Scientometric indicators. Ciência da Informação, v.27, n.2, p.141-148, 1998.

TransInformação, Campinas, 19(2):153-168, maio/ago., 2007 
TRIGUEIRO, M.G.S. Bioprospecção, uma nova fronteira da sociedade. 2006. Relatório de Pesquisa (DPCT- IG) - Universidade Estadual de Campinas.

THOMSON. Web of Science 7.0. Philadelphia: Thomson Corporation, c2004. Disponível em: <http://thomsonscientific. $\mathrm{com} / \mathrm{media} / \mathrm{scpdf} /$ wos7-workshop po.pdf $>$. Acesso em: $15 \mathrm{mar}$. 2006.

VAN RAAN, A.F.J.; VAN LEEUWEN, T.N. Assessment of the scientific basis of interdisciplinary, applied research: Application of bibliometric methods in Nutrition and Food Research. Research Policy, v.31, n.2, p.326-337, 2002.
VELHO, L. Indicadores cientificos: aspectos teoricos y metodologicos. In: MARTINEZ, E. (Org.). Ciencia, tecnología y desarollo: interelaciones teóricas y metodológicas. Caracas: Nueva Sociedad, 1994.

VIEGAS, C.; BOLZONI, V.S.; BARREIRO, E.J. Os produtos naturais e a química medicinal moderna. Química Nova, v.29, n.2, p. 326-337, 2006

ZIPP, L. S. Core serial titles in an interdisciplinary field: the case of environmental geology. Library Resources \& Technical Services, v.43, n. 1, p.28-36, 1999. 